\title{
Nutritional Analysis of Chyawanprash
}

\author{
Suchita Manish Sheth, \\ Associate Professor, Smt. KGMP Ayu College, Mumbai 400002.
}

Author Correspondence: smsheth1972@gmail.com

\begin{abstract}
Ayurveda a great life science provides many Novel concepts like Rasayan,Vajikaran. Chyawanprash, is one of the Rasayan, used as antiageing process and is being used since ages, but still the standardization is lacking , The present study is aimed to standardize Chyawanprash for estimation of Nutritional components present in it by sophisticated analytical tools. The study shows that it contains maximum Iron which is, in fact, more than recommended daily allowances and Fat is less than $0.1 \%$ and other nutritional components as per table 2 . This validates the claim that it can be given to patients with heart diseases. Vitamin $\mathrm{C}$ present in Raw Amlaki was 447 mg/100 gm, which substantially reduced after processing which was reduced to $7.25 \mathrm{mg} / 100 \mathrm{gm}$. Phosphorous detected was $77 \mathrm{gm} / 100 \mathrm{gm}$. There was no presence of heavy metals detected. Hence these parameters and the developed methods may be considered as a tool for assistance to the scientific organization and the manufacturers in developing standards for Chwawanprash.
\end{abstract}

Keywords: Nutritional Analysis, Chyawanprash, Rasayan, Vajikaran, Amlaki, anti-ageing

\section{INTRODUCTION}

Ayurveda the life of science mainly emphasis on wellbeing by achieving youth, vigour and immunity in body by adopting Routine lifestyle mentioned in Ayurveda and intake of certain food supplements which are known as Rasayana ${ }^{l}$.Chyawanprash is one of the Rasayana mentioned along with guduchi etc $^{2}$, which falls under kutipraveshik method for Rasayan intake ${ }^{3-5}$ which imparts immunity, strengthens body, anti-ageing $^{6}$, Aphrodiasic ${ }^{7-11}$. Chyawanprash is used since ages, but the nutritional value is not estimated till date.

\section{AIM AND OBJECTIVES}

1. To incorporate sophisticated instruments and methods for achieving purity of Chyawanprash.

2. To standardize and analyze the inprocess drug with following parameters - Duration, Temperature, $\mathrm{pH}$.

3. To assess the result obtained and to validate the claims made. 
4. To assess nutritional assay for Macro and Micronutrients

3. MATERIAL AND METHODS 12

3.1. Ingredients

Figure 1: Ingredients of Chyawanprash Avaleha (Drugs used for Decoction)

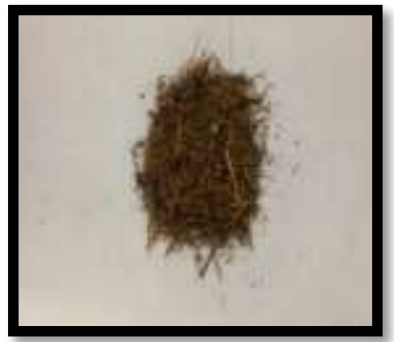

2.1 Patala

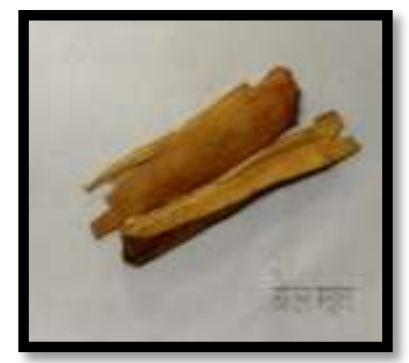

2.4 Bilwa

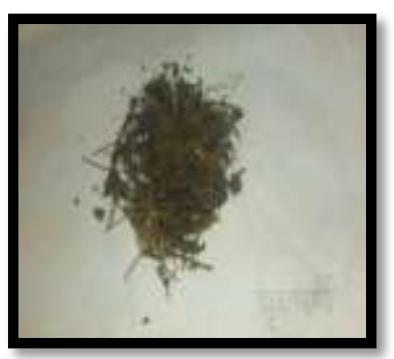

2.7 Mudgaparni

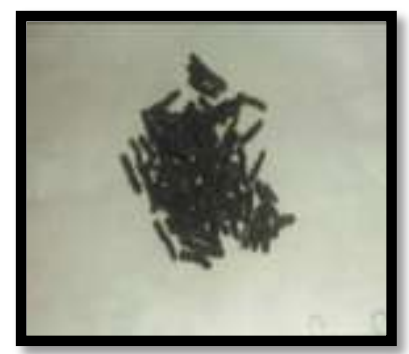

2.10 Pippali

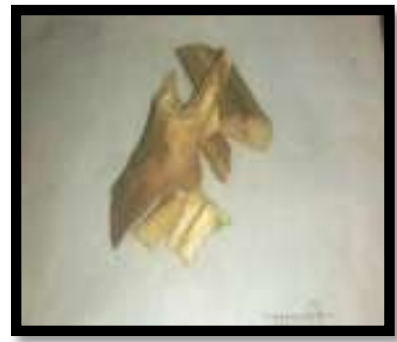

2.2 Arani

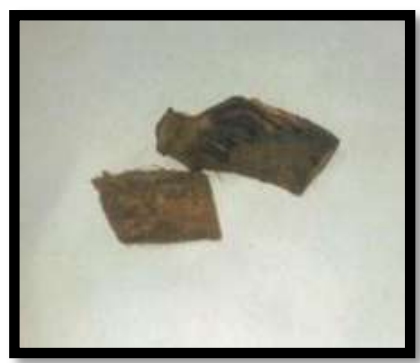

2.5 Arani

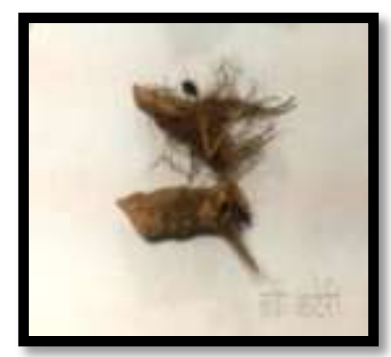

2.8 Mashaparni

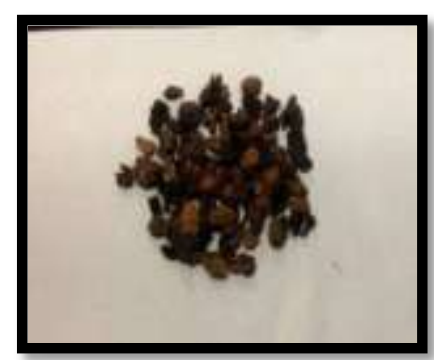

2.11 Karkatashrungi

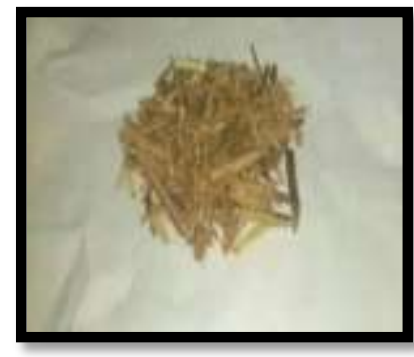

2.3 Gambhari

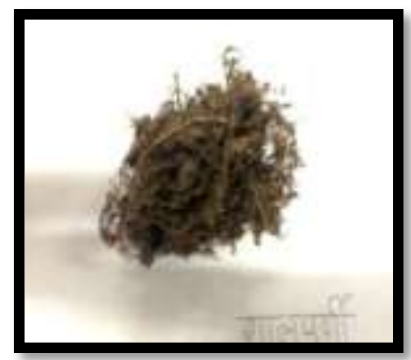

2.6 Gambhari

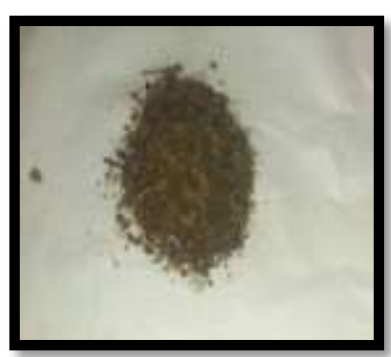

2.9 Kantakari

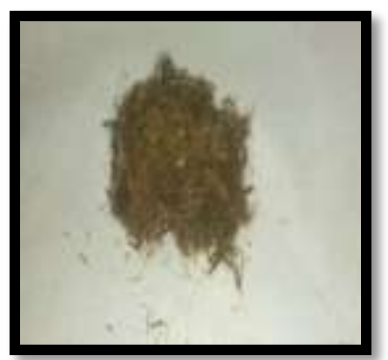

2.12 Bala 


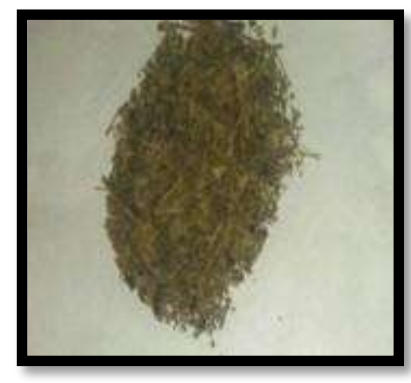

2.13 Bhumyamalaki

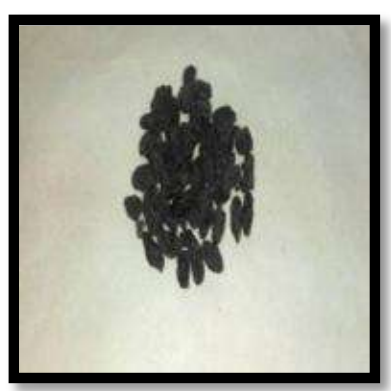

2.16 Draksha

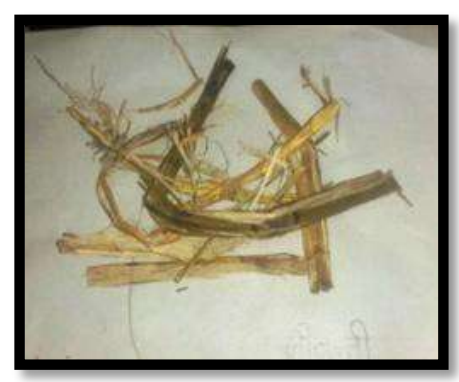

2.19 Jivanti

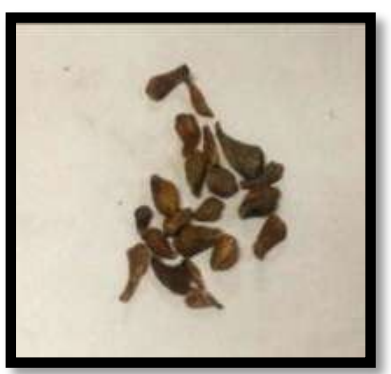

2.22 Rishabhak

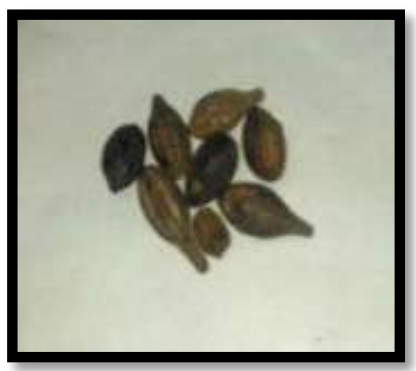

2.14 Haritaki

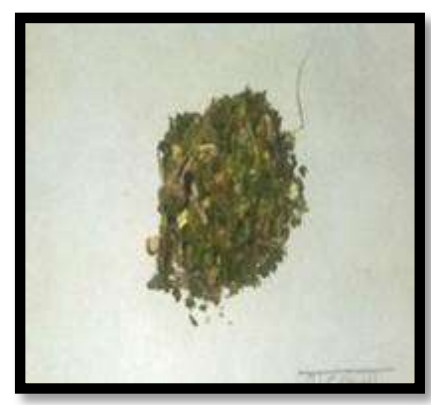

2.17 Vasa

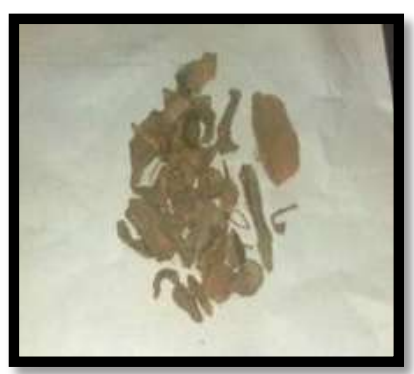

2.20 Karchu

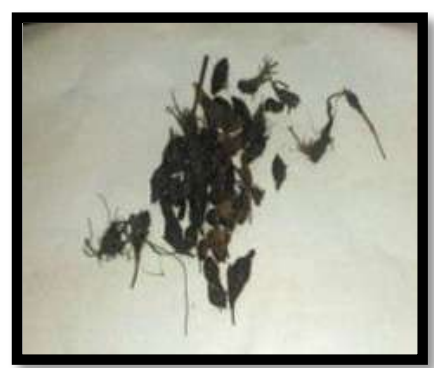

2.23 Musta

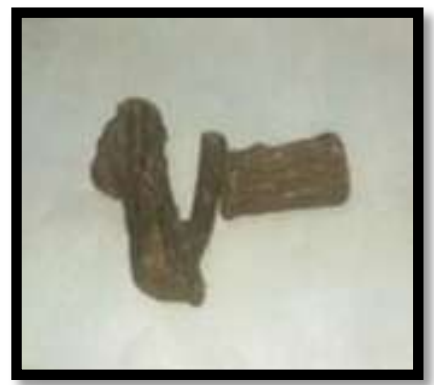

2.15 Guduchi

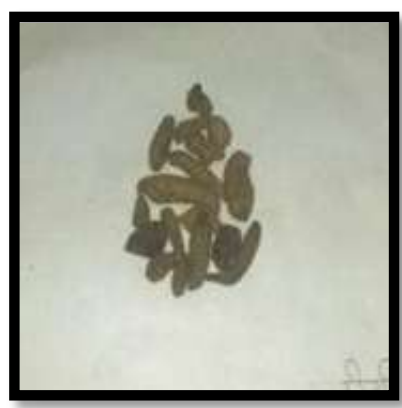

2.18 Riddhi

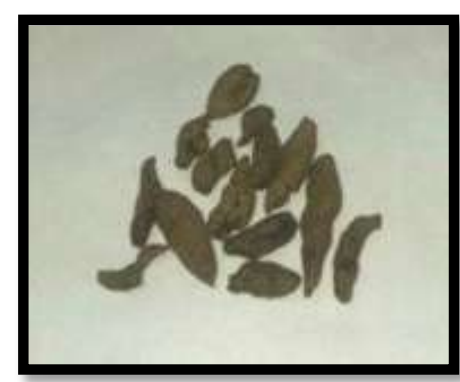

2.21 Jeevak

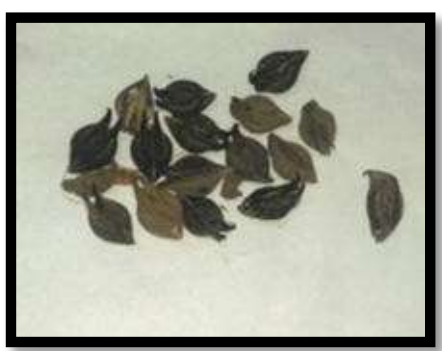

2.24 Kakanasa 


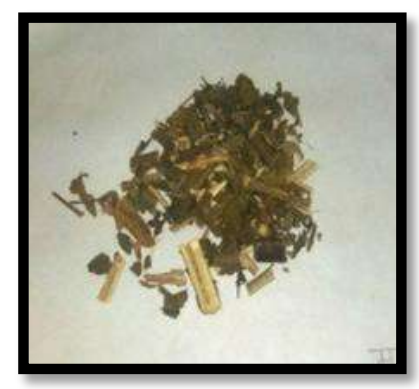

2.25 Mudgaparni

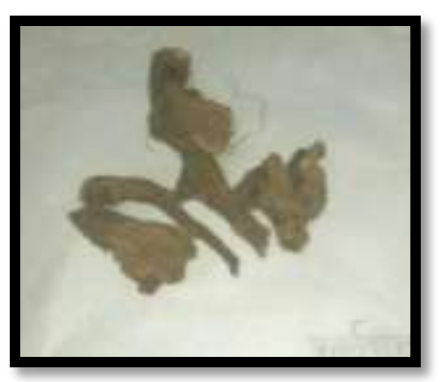

2.28 Punarnava

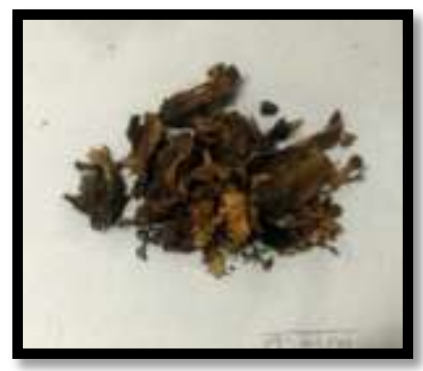

2.31 Kamal

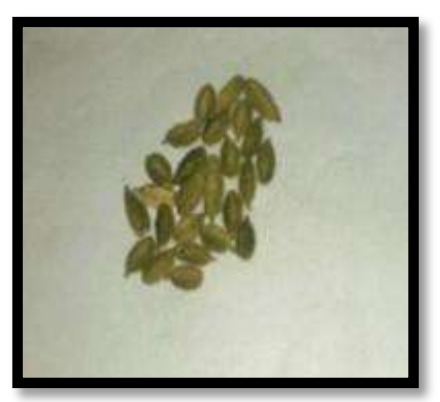

2.34 Ela

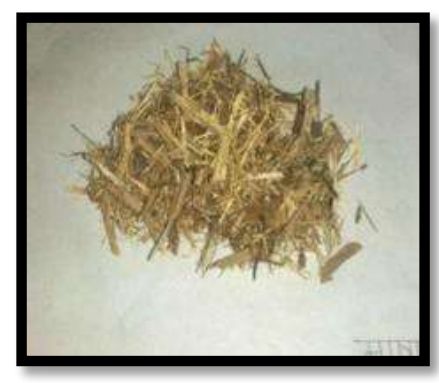

2.26 Mashaparni

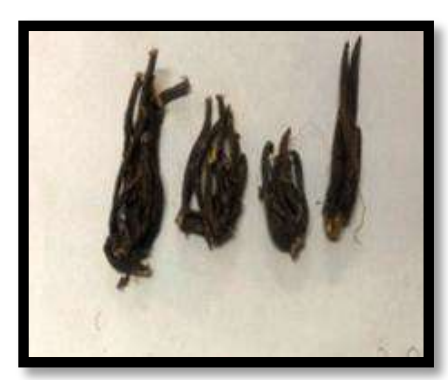

2.29 Kakoli

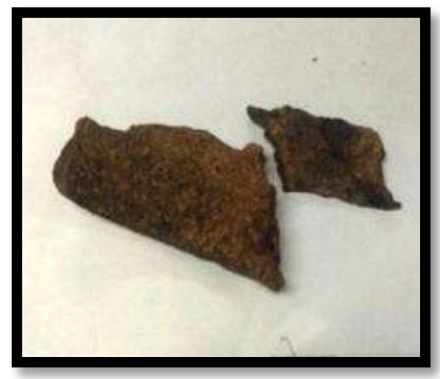

2.32 Meda

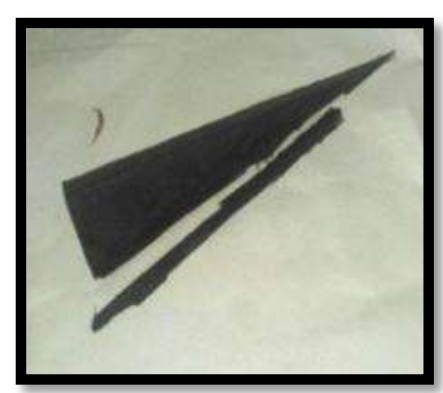

2.35 Agaru

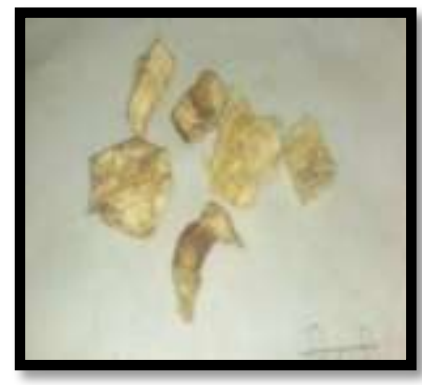

2.27 Vidari

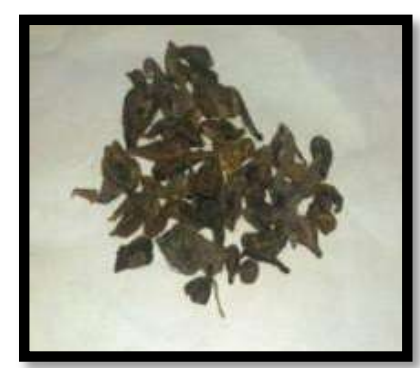

2.30 Kshir Kakoli

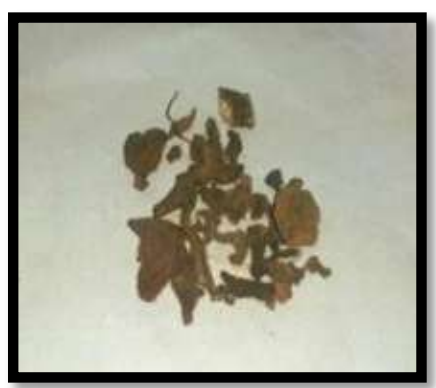

2.33 Mahameda

3.2. Method of preparation 
$5 \mathrm{Kg}$ Amala were weighed and washed. Raw drugs (1824 gm) were weighed and soaked in $12 \mathrm{~L}$ of water overnight. Next day aamla were tightened in a cotton cloth and were immersed in decoction (as in Dola yantra). Then the decoction was boiled till it reduced $1 / 8^{\text {th }}$. Seeds and fibres of cooked aamla were removed and were mashed into pulp. This pulp is then roasted in cow ghee till it leaves oil, at $40^{\circ} \mathrm{C}$ for about $2-3$ hours. Once pulp starts leaving oil, pour decoction and sharkara into it and heat it till avleha consistency is achieved. Finally, powdered prakshep dravyas were added and after cooling madhu was added. The obtained formulation is known as Chyawanprash. Duration for preparation: 4 days, $\mathrm{pH}$ : 3-4.

\begin{tabular}{|l|l|}
\hline Various stages of preparation \\
Bharjana Sanskara (Amalaki \& Goghrita) - \\
Initial Stage
\end{tabular}


3.3. Standardization and Analytical Study:

A. In process standardisation.

B. Finished product- standardisation.

\section{A. STANDARDIZATION OF RAW DRUGS}

Table 1: Results obtained after Nutritional Analysis of Chywanprash

\begin{tabular}{|l|c|c|}
\hline Parameters & Value & Unit of Measures \\
\hline Moisture & 24.26 & $\%$ \\
\hline Ash & 5.21 & $\%$ \\
\hline Protein & $(\mathrm{N} \times 6.25) 1.15$ & $\%$ \\
\hline Fat & $<0.1$ & $\%$ \\
\hline Carbohydrates & 69.28 & $\%$ \\
\hline $\begin{array}{l}\text { Dietary fibres } \\
\text { (soluble \& insoluble) }\end{array}$ & 12.09 & $\mathrm{ug} / 100 \mathrm{~g}$ \\
\hline Vitamin A & 22.7 & $\mathrm{mg} / 100 \mathrm{~g}$ \\
\hline Vitamin C & 7.25 & $\mathrm{mg} / 100 \mathrm{~g}$ \\
\hline Vitamin E & 1.79 & $\mathrm{mg} / 100 \mathrm{~g}$ \\
\hline Calcium & 72.22 & $\mathrm{mg} / 100 \mathrm{~g}$ \\
\hline Iron & 8.14 & $\mathrm{mg} / 100 \mathrm{~g}$ \\
\hline Phosphorous & 76.81 & $\mathrm{mg} / 100 \mathrm{~g}$ \\
\hline Magnesium & 36.79 & $\mathrm{mg} / 100 \mathrm{~g}$ \\
\hline Potassium & 30.10 & $\mathrm{mg} / \mathrm{kg}$ \\
\hline Selenium & Below limit of quantification & $\mathrm{mg} / \mathrm{kg}$ \\
\hline Mercury & Below limit of quantification & $\mathrm{mg} / \mathrm{kg}$ \\
\hline Lead & Below limit of quantification & \\
\hline
\end{tabular}

\section{OBSERVATIONS AND RESULTS} PHARMACEUTICAL STUDY -

Pharmacognostical evaluations shows Dashmool and Ashtavarg which are not genuine still they are being used by traders.
The studydrugs used were identified and authenticate by pharmacognosy and then subjected to study.

B. FINISHED

PRODUCT STANDARDISATION

Chyawanprash was sent for its nutritional analysis at Microchem Silliker (Navi Mumbai, Ghansoli). Result obtained as following:
$500 \mathrm{~g}$ Amala were weighed and washed. Raw drugs were weighed and soaked in $12 \mathrm{~L}$ of water overnight. each Amala weighed $\sim 10 \mathrm{gm}$. Then the decoction of $12 \mathrm{~L}$ was boiled till it reduced $1 / 8^{\text {th }}$ i.e. 1.5 L Amala gets softened.

- $\mathrm{pH}$ of decoction: 4.

- Temperature- Flame while making decoction was: $263^{\circ} \mathrm{C}$, decoction: $120^{\circ} \mathrm{C}$.

- The pulp roasted in butteroil till it leaves oil, at $40^{\circ} \mathrm{C}$ for which duration was about 2-3 hours. 
- Once pulp starts leaving oil pour decoction into it and heat it till avleha consistency is achieved.

- After that finally powdered prakshep dravyas were added and after cooling

\section{TABLE 2 ANALYTICAL STUDY DATA OF FINISHED PRODUCT (CHYAWANPRASH)}

\begin{tabular}{|c|c|c|c|c|c|}
\hline $\begin{array}{l}\text { Sr. } \\
\text { No }\end{array}$ & $\begin{array}{c}\text { Nutrients } \\
\text { analyzed in } \\
\text { chayawnprash }\end{array}$ & $\begin{array}{c}\text { Chyawanprash/ } \\
100 \text { gms as per } \\
\text { analysis }\end{array}$ & $\begin{array}{l}\text { Nutrients/day as per } \\
\text { FDA ranging from } \\
\text { children to adult }\end{array}$ & $\begin{array}{c}\text { Dose in per } \\
40 \text { gms of } \\
\text { chyawanprash }\end{array}$ & Deficient \\
\hline 1. & Protein & $1.15 \%$ & $\begin{array}{l}50 \mathrm{gm} / \mathrm{day}(0.6 \mathrm{~g} / \mathrm{Kg} / \mathrm{d}) \\
\text { i.e. } 10-14 \% \text { of Calories }\end{array}$ & $20 \mathrm{gm}$ & $30 \mathrm{gm}$ \\
\hline 2 & Carbohydrate & $69.28 \%$ & $\begin{array}{l}300 \mathrm{gm} / \text { day } \\
55 \% \text { of Calories should } \\
\text { come from carb. }\end{array}$ & $27.712 \mathrm{gm}$ & $\begin{array}{l}272.28 \\
\mathrm{gm}\end{array}$ \\
\hline 3 & Fat & $<0.1 \%$ & $\begin{array}{l}65 \mathrm{gms} / \text { day } \\
35 \% \text { of Calories should } \\
\text { come from Fat. } \\
\text { Saturated/unsaturated - } \\
>10 \% \\
\text { Poly/monosaturated - } \\
>10 \%\end{array}$ & $0.04 \mathrm{gm}$ & $64.96 \mathrm{gm}$ \\
\hline 4 & Vit A & $22.7 \mathrm{mcg} / 100 \mathrm{gm}$ & \begin{tabular}{|l|}
$500 \mathrm{IU}$ \\
Age 1-8 yrs - 1000-1300 IU \\
Adult - 3000IU (M) \\
2300 IU (F) \\
\end{tabular} & $\begin{array}{l}30.264 \mathrm{mcg}= \\
9.0792 \mathrm{IU}\end{array}$ & $490.9 \mathrm{IU}$ \\
\hline 5 & Vit E & $9 \mathrm{mg} / 100 \mathrm{gm}$ & \begin{tabular}{|l|}
30 IU \\
Age 1-8 yrs - 13-16 IU \\
Adult - 33 IU (S), \\
22 IU(M)
\end{tabular} & $\begin{array}{l}7.16 \mathrm{mg}= \\
7160 \mathrm{mcg}=10.68 \\
\text { IU }\end{array}$ & $20.68 \mathrm{IU}$ \\
\hline 6 & Vit C & $5 \mathrm{mg} / 100 \mathrm{gm}$ & \begin{tabular}{|l|}
$60 \mathrm{mg}$ \\
Age $1-8$ yrs - 25mg \\
Adult - 90mg (M), \\
$75 \mathrm{mg}(\mathrm{F})$ \\
\end{tabular} & $29 \mathrm{mg}$ & $31 \mathrm{mg}$ \\
\hline 7 & Calcium & $\begin{array}{l}72.22 \mathrm{mg} / 100 \\
\mathrm{gm}\end{array}$ & \begin{tabular}{|l}
$1000 \mathrm{mg}$ \\
$700-1000 \mathrm{mg}$ (Children) \\
Adult $-14 \mathrm{yr}-$ \\
$1100 \mathrm{mg} / \mathrm{d}$ \\
$70 \mathrm{yr}-1000 \mathrm{mg} / \mathrm{d}$
\end{tabular} & $300 \mathrm{mg}$ & $700 \mathrm{mg}$ \\
\hline 8 & Iron & $8.14 \mathrm{mg} / 100 \mathrm{gm}$ & \begin{tabular}{|l|}
$18 \mathrm{mg}$ \\
Age $1-8 \mathrm{yrs}-7-10 \mathrm{mg}$ \\
Adult $-8 \mathrm{mg}(\mathrm{M})$, \\
$18 \mathrm{mg}(\mathrm{F})(19-50 \mathrm{y})$, \\
$8 \mathrm{mg}(\mathrm{F})(51+\mathrm{y}), 27$ \\
mg (Pregnant), 9mg \\
(Lactation) \\
\end{tabular} & $32.56 \mathrm{mg}$ & $\begin{array}{l}\text { More of } \\
14.56 \\
\mathrm{mg}\end{array}$ \\
\hline 9 & Dietary fibre & $12.09 \%$ & $25 \mathrm{gms} /$ day & $4.836 \mathrm{gm}$ & $5 \mathrm{gm}$ \\
\hline
\end{tabular}




\begin{tabular}{|c|c|c|c|c|c|}
\hline \multirow[t]{3}{*}{10} & \multirow[t]{3}{*}{ Phosphorus } & \multirow[t]{3}{*}{$76.81 \mathrm{mg} / 100 \mathrm{gm}$} & $1000 \mathrm{mg} /$ day & $30.724 \mathrm{mg}$ & 969.28 \\
\hline & & & $\begin{array}{l}\text { Age } 1-8 \text { yrs }-460- \\
500 \mathrm{mg}\end{array}$ & & \\
\hline & & & Adult - 700mg & & \\
\hline \multirow[t]{3}{*}{11} & \multirow[t]{3}{*}{ Magnesium } & \multirow{3}{*}{$\begin{array}{l}36.79 \mathrm{mg} / 100 \\
\mathrm{gm}\end{array}$} & $420 \mathrm{mg} /$ day & $14.716 \mathrm{mg}$ & $405.28 \mathrm{~s}$ \\
\hline & & & $\begin{array}{l}\text { Age 1-8 yrs - 80- } \\
\text { 130mg }\end{array}$ & & \\
\hline & & & $\begin{array}{l}\text { Adult - 400mg (M) } \\
(19-30 \mathrm{yr}), 420 \mathrm{mg}(\mathrm{M}) \\
(30+), 310 \mathrm{mg}(\mathrm{F})(19- \\
30 \mathrm{yrs})\end{array}$ & & \\
\hline \multirow[t]{3}{*}{12} & \multirow[t]{3}{*}{ Potassium } & \multirow[t]{3}{*}{$\begin{array}{l}302.10 \\
\mathrm{mg} / 100 \mathrm{gm}\end{array}$} & $\begin{array}{l}\text { Age1-8 yrs - 3- } \\
\text { 3.8mg/day }\end{array}$ & & \\
\hline & & & $\begin{array}{l}\text { Adult }-4.7 \mathrm{gm} / \mathrm{d}, \\
5.1 \mathrm{gm} / \mathrm{d}(\text { Lactation })\end{array}$ & & \\
\hline & & & $\begin{array}{l}\text { Age 1-8 yrs - 80- } \\
\text { 130mg }\end{array}$ & & \\
\hline \multirow[t]{3}{*}{13} & \multirow[t]{3}{*}{ Selenium } & \multirow{3}{*}{$\begin{array}{l}\text { Below limit of } \\
\text { quantification }\end{array}$} & 55 microgram/day & & \\
\hline & & & $\begin{array}{l}\text { Age } 1 \text {-8 yrs - 22- } \\
\text { 30mcg/day }\end{array}$ & & \\
\hline & & & $\begin{array}{l}\text { Adult }-55 \mathrm{mcg} / \mathrm{d} \\
70 \mathrm{mcg} / \mathrm{d}(\mathrm{L}) 60 \mathrm{mcg} / \mathrm{d} \\
\text { (Preg) }\end{array}$ & & \\
\hline 14 & Mercury & Same as above & & & \\
\hline 15 & Lead & $0.48 \mathrm{mg} / \mathrm{kg}$ & & & \\
\hline
\end{tabular}

* Institute of medicine of National Academy of Sciences, Nov 30, 2010. Daily Values from NIH, Food labelling guide, FDA, 2013.

The present research drug in $40 \mathrm{gm}$ of sample contains the following nutritional quantity in comparison to Food drug and admission led Recommended Daily Allowance (RDA).

Chyawanprash taken in an advisable dose will match for protein, Vitamin C, Dietary fibre, Calcium deficit for $100 \mathrm{mg}$, Abundance of Iron as per RDA. It is deficit in Magnesium and Phosphorous by $375.8 \mathrm{mg}$ and $907.8 \mathrm{mg}$ respectively.
Potassium was deficit by 2.7 gm. Selenium and Mercury was below limit of quantification and Lead was $0.48 \mathrm{mg} / \mathrm{kg}$

\section{DISCUSSION}

The study entitled nutritional Assay of in Chyawanprash has 3 main sections Pharmaceutical study, Standardization and analytical study .Pharmaceutical study-Chyawanprash was prepared using standard text sharangdhar. Before the preparation of Chyawanprash the raw drug was analysed for pharmacognostical evaluation .Finished product was analysed for the presence of nutritional component in it. The chart below enlists the same. 
TABLE 3 -TEST REPORT OF NUTRITIONAL COMPONENTS OF CHYAWANPRASH

\begin{tabular}{|l|l|c|c|l|}
\hline $\begin{array}{l}\text { Sr } \\
\text { No }\end{array}$ & Parameter name & Result & $\begin{array}{c}\text { Unit of } \\
\text { measurement }\end{array}$ & \multicolumn{1}{|c|}{ Method } \\
\hline 1 & Moisture & 24.26 & $\%$ & IS: 6287-1985(R 2005) \\
\hline 2 & Ash & 5.21 & $\%$ & FSSAI MAN 5 \\
\hline 3 & Protein & $(\mathrm{NX6.25)1.15}$ & $\%$ & AOAC 2001.11 \\
\hline 4 & Fat & 60.1 & $\%$ & IS:6287-1985 \\
\hline 5 & Carbohydrate & 12.09 & $\%$ & AOAC 986.25 \\
\hline 6 & $\begin{array}{l}\text { Dietary fibre } \\
\text { (soluble \& } \\
\text { insoluble) }\end{array}$ & & $\%$ & AOAC 991.43 \\
\hline 7 & Vitamin A & $7.25($ Finished & $\mathrm{mg} / 100 \mathrm{~g}$ & AOAC 2012.22 \\
\hline 8 & Vitamin C & product) & & \\
& & $446.72($ Raw Amla) & & AOAC967.21 \\
\hline 9 & Vitamin E & 1.79 & $\mathrm{Mg} / 100 \mathrm{~g}$ & AOAC 997.03 \\
\hline 10 & Calcium & 72.22 & $\mathrm{Mg} / 100 \mathrm{~g}$ & AOAC 985.35 \\
\hline 11 & Iron & 8.14 & $\mathrm{Mg} / 100 \mathrm{~g}$ & AOAC 985.35 \\
\hline 12 & Phosphorous & 76.81 & $\mathrm{mg} / 100 \mathrm{~g}$ & $\mathrm{MC} / \mathrm{SOP} / \mathrm{INS} / 21$ \\
\hline 13 & Magnesium & 36.79 & $\mathrm{mg} / 100 \mathrm{~g}$ & AOAC 985.35(19 \\
\hline
\end{tabular}

Iron present is maximum i.e. more than recommended daily allowances and Fat is less than $0.1 \%$.This validates the claim that it can be given to patients with heart diseases. Vitamin $\mathrm{C}$ present in Raw Amlaki was 447 mg/100 gm, which substantially reduced after processing which was reduced to $7.25 \mathrm{mg} / 100 \mathrm{gm}$. Phosphorous detected was $77 \mathrm{gm} / 100$ gm. There was no presence of heavy metals detected.

\section{CONCLUSION}

The present work was carried out to assay the nutritional value of Chyawanprash. The result obtained as set to develop standard operating process for method of preparation of Chyawanprash and the nutritional assay of the food components were compared with Recommended daily allowance with that of Food drug and administration. The result indicates the presence of nutrient as per Table 3. Method of food component detection was done by using standard AOAC.
Hence these parameters and the developed methods may be considered as a tool for assistance to the scientific organization and the manufacturers in developing standards. The present work is a small step towards development of standard operating process of Chyawanprash a standard assay of nutritional component present in it.

This will also help to produce uniform standard products, which will restore in the faith of the claims made in Ayurvedic systems.

SUMMARY: yawanprash sample was prepared in the Laboratory according to the standard text. Pharmacognostical evaluation was done by expert. The data analysis revealed most of the materials are not available, instead their alternative herbs are used (Table 2). Chemical analysis was done using various parameters viz fat, carbohydrate, calcium, iron, listed in Table1. The 
chemical analysis for the evaluation of nutritional components showed maximum percentage of iron etc, as per Table 1. In-process standardization showed the duration, temperature $\mathrm{pH}$. All these parameters of Table 1, Table 2 and Table 3 together can be used successfully for analysis of Chyawanprash and can be used as a food supplement required per day, will serve as a basis for fixing standardization parameter.

\section{REFERENCES AND SHORT BIBLIOGRAPHY}

1. Acharya Kashinath Shastri, Charak Samhita; Chaukhamba bharati Academy; Chikitsa Sthana, $19^{\text {th }}$ edition, $1^{\text {st }}$ Chapter, Verse 7, 1993 , p.5

2. Acharya Parashuram Shastri, Sharangadhara Samhita, first part, Chaukhambha Orientalia Publications, $3^{\text {rd }}$ edition, $4^{\text {th }}$ Chapter, Vers 13, 1983. p. 37

3. Acharya Parashuram Shastri, Sharangadhara Samhita, first part, Chaukhambha Orientalia Publications, $3^{\text {rd }}$ edition, $4^{\text {th }}$ Chapter, Vers 13, 1983.p. 37

4. Acharya Kashinath Shastri, Charak Samhita; Chaukhamba bharati Academy; Chikitsa Sthana, $19^{\text {th }}$ edition, $1^{\text {st }}$ Chapter, Verse 7, 1993, p.5
5. Acharya Kashinath Shastri, Charak Samhita; Chaukhamba bharati Academy; Chikitsa Sthana, $19^{\text {th }}$ edition, $1^{\text {st }}$ Chapter, Verse 16-24, 1993, p. 7-9

6. Acharya Kashinath Shastri, Charak Samhita; Chaukhamba bharati Academy; Chikitsa Sthana, $19^{\text {th }}$ edition, $1^{\text {st }}$ Chapter, Verse 7, 1993, p. 8-9

7. Acharya Kashinath Shastri, Charak Samhita; Chaukhamba bharati Academy; Chikitsa Sthana, $19^{\text {th }}$ edition, $1^{\text {st }}$ Chapter, Verse 7, 1993, p.7

8. Acharya Kashinath Shastri, Charak Samhita; Chaukhamba bharati Academy; Chikitsa Sthana, $19^{\text {th }}$ edition, $1^{\text {st }}$ Chapter, Verse 7, 1993, p.6

9. Acharya Ambikadutta Shastri, Sushrut Samhita, Chaukhambha Sanskrit sansthana, Chikitsa Sthana, $8^{\text {th }}$ edition, $27^{\text {th }}$ Chapter, Verse 3-5, 1993, p. 120

10. Acharya Ambikadutta Shastri, Sushrut Samhita, Chaukhambha Sanskrit sansthana, Chikitsa Sthana, $8^{\text {th }}$ edition, $27^{\text {th }}$ Chapter, Verse $3-5$, 1993, p. 121

11. Acharya Anna Kunte, Ashtanga Hridayam, Chaukhambha Orientalia Publications, Uttar Tantra, $7^{\text {th }}$ edition, $39^{\text {th }}$ Chapter, Verse 1-2, 1982, p. 907

12. Acharya Parashuram Shastri, Sharangadhara Samhita, first part, Chaukhambha Orientalia Publications, $3^{\text {rd }}$ edition, $4^{\text {th }}$ Chapter, Vers 13, 1983. p. 